\title{
Position Paper Number 3 for the Workshop "Towards Criteria of Sustainability and Social Meaningfulness in Development": The emerging ecology of genetic, digital and cultural (including non-Western) information environments
}

\section{Susantha Goonatilake}

Royal Asiatic Society, Sri Lanka, susanthag@hotmail.com

$\mathrm{T}$ he environment is usually considered only as the natural namely the physical and biological one. But shifting our focus to the underlying component of the biological environment namely genetic information gives one a different picture. Let us approach this through what is usually considered information.

The global information system is generally understood to be an interconnected net of computers, with sometimes humans interacting with their computer nodes being also taken into account. This network would exchange and process information to arrive at a higher level of processing and cognition, than those of individual constituents, whether machine or humans, would. This describes at a basic level the processes that seem to be occurring due to the rapid development of information technology and its pervasive use. This is a necessary description, but not a sufficient or complete one. This is because the information that would be processed and flow in future networks would not be restricted to those in computer systems alone.

There are other information systems that are increasingly getting interconnected and which would provide the real matrix of the future global meta-information system. These information systems would include not only those in computers but also those in biological systems and cultural systems. These three constitute also three environments. This interconnected whole has not been hitherto taken into account by theorists of the global environment. Yet information in biological systems has been studied by biologists, just as information in cultural systems has been studied by sociologists, and information in computer systems by those in that field.

Incipient now, increasingly in the future, information in the different realms of culture, genes and computers are and would be affecting information flows and processing in the other two realms. Each would be an environment for the other system. So, if one were to consider the global meta-information system as the totality of the complex flows and processing of information in the world, then one would have to consider information in culture, genes and computers as one totality. As the flows and processing of computer information is well described and understood by theorists and students of for example the Internet, I will restrict myself to the genetic and the cultural, describing the latter more fully. Let me elucidate.

\section{Genetic information}

First, is the realm of the natural environment of genetic information, well described by the work of biologists. Over the last three to 4,000 million years, life has evolved to become more complex. This interconnected system is what is usually considered our environment. The evolutionary process in biology is carried through the transmission of genetic information from one generation to another down a lineage. Information is conserved down the line while new information to be transmitted onward is selected - Darwinian wise - when new circumstances arise. This genetic information operates within 
collectivities. In a given reproductive community, there is a large pool of genes. Evolution occurs through selection from this genetic pool, the genetic environment.

\section{Cultural information}

Culture is essentially information that flows through human minds, that is, from one human mind to another human mind. There are also a few species of animals that transmit information acquired through learning from one animal to another. But it is only humans that have the greatest capacity to exchange cultural information and so have an edge in evolutionary terms over the rest of the animal kingdom. It is humans and the outcome of their minds- culture - that interact with the natural environment changing it.

Cultural information is generated within individual human minds in interaction with other humans. Cultural information is exchanged synchronically as say in a conversation in a family or in a more formal setting, say in interactions in an academic seminar. But cultural information is also transmitted diachronically, being passed from generation to generation. Part of the information transmitted to a new generation is kept as memory; while a new generation may modify part of the transmitted information adding new elements while excising out some older information.

Thus, in small groups such as say hunter-gatherers in the Amazon, the store of cultural information of how to deal with the environment that had been transmitted from earlier generations is maintained. However, the hunter-gatherer always samples and tests his environment continually, (as well described in his search for medicinal plants), modifying the information handed over to him. He then transmits that information onwards to the coming generation. When the group shifts to a new environment, it will add new information and excise some old and pass the new totality onwards to future generations. This new information fitting into a fresh environment would have large cores of the old information from its earlier environment. Such core information could well be traced to a long distant past. Cultural information fitting into different environmental niches define particular subcultures, which are collectivities of carriers of common cultural information, different microenvironments within the larger cultural environment. Given sufficient isolation of such sub cultures - micro-cultural environment, different pockets of culture will arise and attendant different lenses of cultural cognition, that is, the different isolated groups will socially construct different cultural realities. The transmission lines of a common culture traced through such groups diachronically will constitute lineages of information in much the same way as do genetic information transmitted through species.

These lineages of cultural information are discerned not only in the flow lines of small tribal groups, which are socially undifferentiated but also in more complex social formations such as those of modern societies. In the latter, social differentiation occurs, for example, according to class and further sub-differentiation occurs on occupational and trade criteria. Consequently, one has macro cultural transmission lines carried forward to future generations along classes and along occupational categories. Quite often such broad bands of cultural information are associated with particular worldviews expressing particular cultural environments. Hence classes are associated with particular sets of consciousness and worldviews, which their individual histories have given them. So do different professional and disciplinary groupings have different worldviews. Such bundles of culture - micro-cultural environments - and their associated worldviews have been extensively studied by social scientists under, for example, social cognition, social construction of reality, ideology and evolutionary epistemology.

Over long time spans, the growth of subcultures' micro-environments and associated lineages is a form of information speciation like that occurs in biology. The result is the increasing complexity of society and increasing complexity in the collectivities of cultural information of systems, of cultural environments. The growth of scientific knowledge within demarcated disciplinary 
boundaries and their speciation into subdisciplines has being well studied by sociologists of science, especially through the school of the social construction of science. Disciplines and their associated knowledge develop within particular social environments as they tackle current problems. The social environments of laboratory groups, "invisible colleges", networks of practitioners and theorists and gatekeepers such as editors of journals each a micro-environment - influence the development of this information. When such knowledge change and differentiate, disciplines emerge together with accompanying new knowledge. With time and associated change, disciplinary lineages split and speciate, as do all cultural lineages.

These information collectivities of environments in the genetic and cultural, now have another information set in the form of information in computers. Computing devices - only somewhat over half a century old - are multiplying and growing in complexity at an exponential rate. Earlier, they were tightly controlled by human programs, now several techniques such as genetic algorithms and neural networks allow computer systems to learn and change their behavior in non trivial ways. Today possibly trillions of the smallest computers interact performing control functions in houses, cars and other devices and so on. These are in addition to more sophisticated and more complex computing entities. An environmental information cloud has been created through such computers.

\section{Hybridization}

These three information realms, of different environmental clouds, - the genetic, the cultural and computer - (that is "artefactual" information) although nominally separate are increasingly hybridizing themselves, merging their information stores and means of processing information - a merging of their different environments. This is occurring through advances in biotechnology and information technology. And these developments will have deep repercussions on all discussions of the global meta-information system, because what will now be interconnected are systems of information, of information environments, which are hybrids of genetic, cultural and artefactual information. Let me explain this merging/hybridization. I have described some of it elsewhere (Goonatilake, 1993; Goonatilake, 1999). The environment for one information system becomes the transaction field for the other. The environment for humans is no longer the natural, of nature not just humans but all three.

Let me begin with the merging of genetic and cultural information. A scientist isolating a gene and splicing it into an existing genome and so creating a new biological entity is doing a cultural act. He takes cultural information, (in this case scientific information on how a gene is held together, how it can be removed and reinserted into another organism) and combines this with genetic information. It is not only scientific information that gets merged as cultural information but also cultural choices such as, is it useful or desirable on medical, ethical, political and economic grounds to introject the particular gene.

Biologists have identified two types of genes, structural genes which build particular aspects of an organism and regulatory genes which control the process. The new set of cultural information which the biotechnologist brings is another control element like regulatory genes on how a set of genes can operate upon. Consequently, cultural information gets merged into the genetic.

The reverse process of genetic information getting merged into cultural information also occurs. Future genetic intervention can change the whole apparatus, namely the brain and the nervous system, through which cultural information is captured and processed. Such genetic interventions can potentially change our sensory inputs, for example getting the ears to capture a different audio spectrum than we are used to. It can also change the relationships of the various parts of the brain thus intimately changing the output of cultural information at a biological level. The two environments the biological and the societal mutually interact, like two interpenetrating clouds. 
And in a further mode, cultural information and artefactual information also merge in a two way process. A person operating a computer, say even a simple word processing program receives its output and so changes his thoughts and behavior. His store of cultural information is changed. Generally speaking computer technology is transforming our imagination. And when a person operates the computer, he in turn transfers elements of his cultural information store to the computer realm. With Artificial Intelligence techniques, such two-way mergings can be non trivial. If the computer is using learning systems like genetic algorithms, fuzzy logic or neural networks, the computer system's internal processes are not directly and/or immediately accessible to humans. The machine environmental clouds interact with the societal/cultural clouds and vice versa.

Artefactual - computer - information and biological information also gets merged. Work on the Human Genome Project and similar projects are so computer intensive that one has to think of it only as a partially merged system. In the opposite direction, bio chips - computer chips with biological elements already built-in also results in merging. There is also an indirect form of merging through computer techniques like neural networks and genetic algorithms which mimic biological systems in computing processing. Some newer approaches use techniques borrowed from immune systems in biology. The machine environment and the biological environment merge.

Often the mergings are not just two-way but three-way, all three environment realms merging. Thus when work is done on the many genome projects around the world, including that of the Human Genome, some of the analysis of the information will be done in the computer mode because that is where the data resides. This then becomes a manipulation jointly of cultural information (from the scientist working on the data), artefactual information (resting in the computer) and of genetic information (from which is extracted the computer data). There are many other three-fold hybrids, and fresh ones are emerging daily. The three environment clouds, - biological (natural), human/cultural and machine/computer - now interact intimately.

These merging processes will result in hybrid micro-environment entities populating various niches in the world. With exploding communication links, they will exchange information intensively and so constitute the emerging global network, the real metainformation environment.

There are already numerous illustrative examples of such hybrid environmental systems nodes in the inclusive metaenvironment environment.

The growth of ubiquitous computation and connectivity promises an all-pervasive system and all pervasive environment where one forgets that it is there. It would be like a jungle in which we would be embedded in an interconnected world. It would have both data and programs in a redundant form very much like the human brain. Ubiquitous computing would thus appear unobtrusive, normal and natural. These systems would connect millions of devices from large supercomputers to cubic-millimeter size "smart dust". In such an emerging world, computers would be embedded in everything and computation would never cease. In such a system, machines would be continuously communicating with each other while human to human communication will only be a very small part of the traffic. The every day human environment as say in furniture or walls would have logic devices, processors, sensors, actuators, communicators, memory systems, cameras and microphones. This would be a seamless environment of computing and communication, of a metaGaia. The system would also learn and adapt, evolving in the process. This would be a merging of micro-environments in the human and computer systems.

\section{Communicating micro- environments}

Therefore, in the emerging world, there would be a multiplicity of processing and communicating environments. These environments could either be of one form of information, that is, either cultural, genetic or computer form, or combinations thereof, either twofold combinations or threefold 
combinations. The exchange of information across these environments would quickly supersede those between and within human communities. Already in the artefactual mode, there are vast amounts of data sloshing around the globe which rival in range those of human communications unmediated by artifacts. Each such hybrid environment would be dynamic and changing, most probably learning according to changes in their environments.

The presence of so many varieties of information processing and communication environments will transfer the concept of a communicating person to a wide variety of cultural, biological and artefactual hybrid environments. Such entities being communicating and intelligent ones, will encroach on the issue of what it is to be a communicator or person in a legal sense.

These all relate to the issue of what constitutes a community or a microenvironment in the new dispensations. In a community, in a micro social environment, members communicate with other members who constitute their "significant others" and thereby change their information states leading to changes in their internal mental behavior and outward external behavior. Because of the multiplicity of new merged hybrid entities that would act as environments in communication, the concept of significant other will now extend beyond humans (and their culture) to genes and artifacts and combinations thereof. In the future, all three environmental realms, will be communicating intensely with each other and their respective hybrids. For all purposes therefore, the system has to be considered as one composite whole of merging environments.

This macro or meta communication system will dwarf in quantity and intensity the existing communication modes. In such a merged system, information is ferried from say culture to gene and then to artifact and to various permutations and combinations of these three realms.

But what cultural information, of what cultural environment, will feed this emerging matrix. Hitherto it had been largely Western cultural elements that would have got embedded in this merged system. But at the present there is a shift of the world's economic axis to Asia, which could have profound implications for the metaenvironment in that the Asian cultural environment could be a major factor in the mix.

\section{The shift to Asia}

Our new century will be marked by several dramatic changes that will leave their effect on knowledge, science and technology. One would be the shift of the centers of gravity of the world in economic and political terms away from its present Eurocentric moorings to Asia, a process that will occur amidst pervasive globalization.

This economic shift is occurring with a re-division of work. It replaces the earlier relocation of brawn work towards cheaper Asian countries with a new relocation of brainpower, and a shift in science and technology towards the Asian cultural region. Thousands of Asians today man research facilities in the West symptomized by the large number of South and East Asian graduate students in American universities. A considerable proportion of scientific personnel in Silicon Valley, the initial breeding ground of information technology are Asians. And in the opposite direction towards Asia software production has emerged as a major growth point in India paralleling the growth of computer hardware in South East and East Asia in the earlier years.

\section{But, is this all there is to it?}

This shift of IT in particular and science and technology in general to Asia is occurring amidst a shift in less measurable cultural spheres where the earlier hegemonic blanket of Eurocentricism is being lifted. Thus some of the best novelists in English, the primary language of globalization, are today those of Asia. Designers from Hong Kong and Japan set clothing fashions. And, Asian cities are the capitals of the cartoon film world. Can this creativity be shifted to IT.

Such Asian inputs become more vital in the case of the newer information 
technologies - information technology and biotechnology - that are more socially responsive than the earlier ones. They can be "cut" and shaped socially in many more and variegated ways than the earlier technologies. As the two technologies are very flexible to social and cultural pressures to their cultural environments, the question then would be which cultures' and which society's values will be mapped within these technologies as they further unfold in the future.

A great Asian imprint on science and technology could come from Asian answers to some of the troubling questions raised by these new technologies. The latter put doubt on some of the most cherished selfperceptions of humans. Biotechnology raises key questions on traditional concepts of what it is to be a living being, including what it is to be uniquely human, biologically. On the other hand, information technology, especially Artificial Intelligence (AI) in mimicking human mental processes, raises questions on what it is to be uniquely human in a cultural sense. A real Asian imprint would require searching answers for these within Asian cultural environments.

Because of such key questions, debates on ethical and cultural issues are shaping both technologies. Thus, releasing of biotechnological products to the atmosphere has been debated within a framework of its potential impact on other organisms. And, advances in medicine relating to say the onset of life and its termination have been hotly discussed and have influenced conventional medical technology. Developments in the new biotechnology stretch these questions very much further, raising fresh and very complicated ethical issues. These discussions and controversies in the cultural and social environment influence and continuously shape the new technology. When one speaks of biotechnology and information technology as I do below, it should be noted that elements of one is seeping from to the other as illustrated by gene chips, bio-chips and sequencing machines. In such situations the boundaries of biotechnology and IT is getting blurred.
However, the social and medical implications of biotechnology have as yet been largely discussed only in Western cultural environments. These debates have unfolded within a context that assumes as universal, Western cultural and social givens, the imprint of the West's religious traditions for example being unconsciously brought in. In Asian countries there has been little debate on these matters. Yet, workers in the field have pointed out that Asian traditions could well give different answers to these questions as for example reflected in the Japanese response to definitions of clinical death

Advances in biotechnology including gene therapy and aided by IT could reshape and reformulate among others, life, death, health and beauty. The ethical as well as esthetic criteria on which these are decided upon are deeply culture bound and if debated within the Asian region's different cultural environments would give different answers from those of the West. And this act of shifting to a different cultural environment would tend to give different directions to the technology.

Advanced information technology especially Al related ones, aims at cloning the partial behavior of the mind. This again would raise deep questions for those parts of Asia that have strong cultural and religious traditions emphasizing the importance of the mind and mind culture. Asian inputs into debates on the ethics and nature of Al could also strongly influence the direction of information technology.

In addition to these specific issues relating to the two new technologies, the Asian region is also rich in non-European derived intellectual activity including aspects of technology and the sciences. Some recent research indicates that these indigenous Asian aspects could also become a useful adjunct to developing new technology in Asia. Let me point out the example of the knowledge elements that are held by presumably the simplest of societies say, hunter-gatherers which are spliced into the modern biology corpus.

The new scientific ways of knowing the world were built up initially in Europe from 
the assembly of cultural elements from within Europe itself as well as from those transmitted to it from outside. Since then, external contributors to this stock of knowledge have diminished. Yet, useful extra European knowledge still exists as exemplified by "lost" knowledge in regional civilizations and say, ethno biology used in search for new medicines. Bringing in knowledge to the dominant knowledge tree can be accomplished in two broad ways, one is to splice-in directly existing material that has demonstrable direct validity. The second would be to bring as metaphors, elements from other traditions that could nudge the imagination and give rise to new concepts. The first attempt would be like the splicing-in to the European tradition of say the knowledge of the compass, gunpowder, or mathematics and knowledge of flora and fauna that occurred during Renaissance and immediately after. The second attempt would be like the many transfers of metaphors that have been constantly used in building the scientific enterprise. I have surveyed such possibilities in a book.

\section{Social theory for new technologies}

The march of science since the Renaissance challenged several held beliefs. In the western world this included a questioning of the anthropocentric world derived from Judeo-Christian roots of Creation. Significant challenges were those of the Copernican Revolution, the Darwinian evolutionary system, and the discovery of the unconscious, none of which one should note would note would not have challenged Asian psyches to the extent they did in the West.

In the new world of information technology and biotechnology there are ethical challenges that have not been met before. These belong to the class of problems raised by the technologies where parts of the body and the mind are cloned. These dilemmas are the subject of intense discussion on the essential nature of the human that is being intruded upon by these technologies. The ethical and conceptual knots of the future brought about by these new technologies could have many uses for
Asian perspectives. Different answers could result.

Apart from lineages in subjects dealing directly with technology related matters, there are lineages and vast store houses in the humanities and philosophical traditions that could feed the cultural inputs and debates on science and technology. South Asia for example is one of the largest store houses of literature in the world. Apart from works in the two classical languages of Sanskrit and Pali, there are large collections in the regional languages. Part of this humanistic literature consists of a large number of allusory texts, replete with perhaps the largest store house of metaphors. If one wishes to catch the imagination roaming in controlled free fall, this, and its relations is one literature to jump into.

The act of scientific creation rarely takes the form of consciously transferring metaphors from one realm to another. The process takes place naturally, subconsciously, although clearly the approach can be cultivated. In such an unconscious mode, a Copernicus transfers the metaphor of a circle intuitively into the movements of the heavens. Later, he checks them against his figures. Once the geometrical pattern is given, and the Gestalt switch made, a Kepler can come at a different time and refine the broad Gestalt into an ellipse.

If one were to do the metaphor searching game unconsciously, then one would have to do it while being immersed in the South Asian culture. A person who is immersed in the culture, and is not restrained to think through his culture, taps the store house of metaphors without conscious effort. But, unfortunately, South Asians themselves doing science have been socialized completely into the western frame. They have - to use the title of an earlier book of mine - Crippled Minds. They tend not to think in a free-floating, unconscious manner. They are always inclined to look over their shoulder about what their mentors in the west say, or would say.

But those unconscious South Asians who do think on their own, can come up with 
major breakthroughs. The classic example is the Indian mathematician Ramanujan, who was "discovered" by the British mathematician Hardy, and brought to England earlier in the $20^{\text {th }}$ century. Ramanujan is today considered one of the greatest mathematicians in the last century as an American book - (Robert Kanigel, The Man Who Knew Infinity: A Life of the Genius Ramanujan, Charles Scribner's Sons, New York, 1991) - readily testifies to. Mathematicians are still poring over his notebooks to dredge for the nuggets he had discovered. But Ramanujan did not follow an imitative Western method. A man fully rooted in his peculiar traditions, he would believe that his results came up on the tongue of his god Narasinha. His was one clear example of unconscious tapping of his cultural environment.

Yet, even without unconsciously tapping the metaphorical database of a civilization, one can consciously train oneself to do so. So can also an outsider to a culture, train himself to be empathetic to a culture and tap its roots. The case of Schrodinger's acknowledged debt to Vedanta philosophy for his quantum physics is well known. The metaphorical uses of non-western sources are virtually limitless in their potential. Only the human imagination can put brakes on to its possible uses. Metaphors are an added tool in the arsenal of indirect approaches to mining the civilizational knowledge and information environment.

The worlds of ideas were brought together and packaged in Europe in a fresh vigorous amalgam. The Renaissance, the Scientific Revolution, the Enlightenment and the great discoveries in the 19th and 20th centuries have been the resultant outcomes. Now, a new packaging is possible. A new historical moment has opened up where the world is increasingly being interconnected in a pervasive spread of globalization. As a result, no single cultural micro-environment can in the future act as a clearing house for all the world's knowledge. The role of a dominant center over a periphery is eroding as near-instant communication begins to spread information and knowledge creation across a wide network. Globalization is opening up centers of dominance away from one central micro-cultural environment to a shifting web (sometimes resident as moving denizens on the Internet). Academic groups are spreading across the world. Further, new historical research is coming up across the world, bringing out hitherto cognitively hidden nuggets.

Inputs into science and technology from different civilizational environments could occur because of this globalization. Already mainstream R\&D is getting relocated for considerations of cost in some cheaper Third World countries like India. Another sciencerelated technological activity, software production has got relocated for similar reasons, and India's software production today rivals many developed countries. Globalization is thus opening up to different cultural environments. Consequently, it will suck up cultural elements from the different global cultural environments and "remarket" them to global niches. Already serious discussions have been held by down-toearth programmers and IT personnel in India of how to tap local cultural sources and so have an edge on the global market (See for example the conference site "Doors of Perception")

The possibilities of enlarging the existing knowledge environment by tapping into civilizational stores are potentially vast. So what is the image of Asian science and technology that we are left with, considering the new tendencies visible on the horizon. Entwined in an increasingly interconnected global environment, the meta-connected merged environment would be molded by different social, cultural and ethical factors, including indigenous Asian elements, as no previous knowledge systems or technologies have been.

\section{Jungles and oceans of information}

The presence of so many varieties of information processing and communication environments will transfer the concept of a communicating 'person' to a wide variety of cultural, genetic and digital hybrids, to hybrid environments. Such environments being communicating and information processing ones will encroach on the issue of what it is to be a communicator or person in a legal 
sense. But this is not the first time that nonhumans have been recognized as legal entities. Laws provide corporations, municipalities and labor unions for example to be for all purposes considered a person in the judicial sense. Such a corporate entity can sue and be sued.

These all relate to the issue of what constitutes a community and social in the new dispensations. In a community, members communicate with other members who constitute their "significant others" and thereby change their information states leading to changes in their internal mental behavior and outward external behavior. Because of the multiplicity of new merged hybrid environments that would act as nodes in communication, the concept of significant other will now extend beyond humans (and their culture) to digital to genetic microenvironments and combinations thereof. In the future, all three realms, will be communicating intensely with each other and their respective hybrids. For all purposes therefore, the system has to be considered as one composite whole, of different environmental clouds interpenetrating.

This macro or meta communication environment will dwarf in quantity and intensity the existing communication modes. In such a merged environment, information is ferried from say culture to artifact to gene and to various permutations and combinations of these two realms.

Let me explore through the human angle how this potent mix of communicating hybrid environments spread around the world will develop. In such a hybrid interconnected system, a person's thought, a figment of his memory, a dab of paint or a soft tune could be converted into a biological organism or computer artifact or combinations thereof. And in the world where computing artifacts could be directly controlled by thought, thought patterns could directly be transferred in godlike fashion to life forms. Within such an extreme extrapolation, one plays music or dance and these get transformed into the music and dance of the genes and so a myriad life forms. Similarly, across the entire merged environment, one could have scintillating points, information tornadoes located at micro-environments in the global meta-information environment from which, issue new bursts of information. Many of these micro-environments would be learning systems reacting to inputs from their meta environments, the reactions depending on their individual make up and histories.

Moving around in this information jungle evokes a comparison with exploring the jungle by those culture/genetic hybrids namely forest dwellers. The latter are immersed in a tangled environment of information extruded - phenotypically - by thousands of plant and animal organisms that surround them. They would sniff around and explore only one or two, ignoring the bulk. So is it, in the new jungle of learning. Exploring hybrids would hunt out for new information that their histories suggest are interesting, while ignoring other information carriers.

Another image that can be evoked to describe the emerging situation is of an ocean of environments- the genetic, the cultural and the artefactual that exist by themselves or in combination. Here currents and bubbles of information are generated. They rise and fall and circulate due to dynamics within each micro-environment as well as from outside it. Because of the ability of information in one system to be translated into another, subterranean currents arising from one mode could influence another. Thus within a human social structure could arise a fresh cultural entity, say a new set of thoughts and this would get expressed as a genetic or artefactual entity. Similarly biological information or artefactual information generated from within their entities will create new hybrids that traverse up and down the hybrid environments.

Across all these three realms there are processes of localization - of clusters being formed - and of globalization, of larger level communicating patterns. In the process, there would be a continuous system of organization of the communicating entities into micro-environments and communities. Such organizations would differentiate the systems in the communicating ocean in three-dimensions, upwards, downwards and sideways. The environmental ocean will be 
interconnected by connecting channels. Sometimes a quickly formed hybrid may dive bomb into this mixed ocean and carve out a new pathway. Sometimes in the process, existing pathways will be eliminated. The result will be a dynamic bubbling ocean, a truly all-inclusive global meta-environment system. A new global community, a new meta-environment, is in the making.

\section{References}

Goonatilake, S. (1993). The New Technologies and the 'End of History'. Futures Research Quarterly, 9(2), 71-93.

Goonatilake, S. (1999). Merged Evolution: the Long Term Implications of Information Technology and Biotechnology. New York: Gordon and Breach.

Kanigel, R. (1991). The Man Who Knew Infinity: A Life of the Genius Ramanujan. New York: Charles Scribner's Sons.

\section{About the Authors}

Susantha Goonatilake

Susantha Goonatilake is the President of the Royal Asiatic Society, Sri Lanka. [email: susanthag@hotmail.com] 